\title{
The revision of the categories of mood, anxiety and stress-related disorders in the ICD-11: a perspective from the Arab region
}

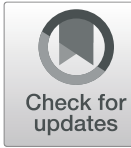

Joseph R. El Khoury, Evelyne A. Baroud and Brigitte A. Khoury

\begin{abstract}
Background: The World Health Organization estimates that $75 \%$ of patients with mental and substance use disorders in low- and middle-income countries do not have access to the care needed. In the Arab World, approximately 100-140 million people suffer from at least one psychiatric disorder. One main criticism of classification systems has been their lack of cultural sensitivity. The International Classification of Diseases, 11th edition aims to improve clinical utility of psychiatric classification across cultures. Mood, anxiety and stress-related disorders are the most common psychiatric manifestations and the most impacted by cultural factors.

Main body: Relying on rigorous field testing, including in three Arab countries, clinically meaningful changes have been introduced in the International Classification of Diseases 11. These include new disorders such as complex post-traumatic stress disorder and prolonged grief disorder. In classifying mood episodes, the pattern of symptoms over time is emphasized. Disorders associated with anxiety provide the basis for a new grouping separate from obsessive-compulsive-related disorders. Lastly, culture and its impact are incorporated into each diagnostic grouping.

Conclusions: This latest version of the International Classification of Diseases prioritizes addressing gaps in the validity and reliability of psychiatric classification. The methodology adopted in this latest revision is encouraging and opens the way to truly global collaboration on refining psychiatric diagnoses and practice.
\end{abstract}

Keywords: ICD11, Mood disorders, Anxiety disorders, Stress-related disorders

\section{Background}

The World Health Organization (WHO) is responsible for setting the global standard of classification for all medical disorders, including mental disorders. The International Classification of Diseases (ICD) is the official diagnostic system across WHO member states for the collection and recording of health statistics [23]. Despite the predominance of the Diagnostic and Statistical Manual of Mental Disorders (DSM) published by the American Psychiatric Association [1] in academic and clinical spheres, the US is no exception to this rule [50]. The past revisions of the ICD and DSM have consistently produced increasingly complex classifications that are not necessarily clinically relevant in most

\footnotetext{
* Correspondence: bk03@aub.edu.lb

Department of Psychiatry, American University of Beirut, Beirut 1107 2020, Lebanon
}

settings. Many patients present with conditions that do not fit any of the available diagnoses. Many others are diagnosed with more than one condition. A third group will have their initial diagnosis revised by the same clinician or challenged by another, often on a subjective basis. The stability of psychiatric diagnoses remains poor for a wide range of conditions $[4,11]$. Culture not only influences the patient presentation but also the psychiatrist's perception of symptoms [7].

The last version of the ICD was the 10th edition, adopted in 1990 [67] by the world health assembly at WHO. Consensus in the international psychiatric community had been in favor of a simpler system of fewer than 100 categories [64]. The latest revision process prioritized addressing gaps in the validity and reliability of the current diagnostic systems as well as in the initial utility of the classification, making it hence an easier and more applicable tool to use by clinicians around the 
world [23]. The importance of clinical utility is closely related to the diagnosis as a way to inform the choice of treatments and interventions. Currently, treatments appear to work within and across diagnoses without distinction; one such pharmacological example would be the increasing use of atypical antipsychotics in the treatment of mood and anxiety disorders [8]. Another example is the similar effect sizes for medications across anxiety disorders [5]. This is paralleled at the level of psychological interventions in the generalization of the application of cognitive behavioral therapy to most mental health conditions, including post-traumatic stress disorder, obsessive-compulsive disorder, panic disorder, generalized anxiety disorder, social anxiety disorder, and specific phobias [33] as well as depressive disorders [16].

The overwhelming majority of mental health care globally is delivered by non-specialists, such as primary care workers, particularly in developing countries [48]. Over the last decades, mental health diagnostic classification systems have produced versions that are, in theory, better suited to the primary healthcare context. The International Classification of Diseases, 10th edition (ICD-10) Primary Health Care Version (ICD-10 PHC) published by the WHO is the primary care version of the ICD-10 dedicated to that purpose [63]. In practice, despite this effort, doubts remain over their level of utilization [6]. Further engagement of primary care workers, whether from a medical background or from other disciplines, in the development of new classification systems is desirable [48]. This was the methodology used in the revision of the ICD-10 while adhering to the established rules in general medical classification [3].

In order to revise the meta-structure of the ICD, the various working groups, made of a range of experts in the field, drew on empirical evidence from studies and relied on a methodology already tested in other disciplines. A study including over 1000 psychiatrists and psychologists from 64 countries revealed consistent conceptualizations of classification along three main axes: internalizing vs externalizing disorders, disorders of development vs those of adult onset, and those of functional etiology vs an organic one. This meta-structure, implemented for International Classification of Diseases, 11th edition (ICD-11), was deemed a better fit than its counterpart in ICD-10 and the latest DSM version [52]. In particular, the ICD-11 lists essential features of diagnoses rather than criteria for diagnoses, without a requirement for specific numbers of symptoms or time frames, unless there is strong cause for their incorporation or there is empirical evidence that they are present throughout countries and across cultures [51].

One guiding principle of the revision process was the clinical utility of the classification in terms of communication value for clinicians, administrators, and service users at clinical but also at policy level [23]. This has implications for resource management and intervention design within specialist and generic settings globally; the ICD-11 is therefore intended as an open accessible global resource that may be used not only by clinicians but also by researchers, policy makers, administrators, governments, as well as patients and their advocates [23].

According to the World Health Organization, it is estimated that around $75 \%$ of patients who suffer from mental, neurological, and substance use disorders in low- and middle-income countries do not have access to the care they need [70]. The ICD revisions go hand in hand with advancements in global mental health, which aim to reduce the treatment gap that reaches up to 85$90 \%$ in such countries [54]. The discrepancy between the high burden of mental disorders and accessibility to evidence-based services is significant in low- and middleincome countries, where for example only one out of 27 persons with depression receives treatment [61]. Therefore, advancing mental health care faces the limitations of policy making, legislation, and resources [49].

The ICD revision working groups delegated by the WHO prioritized the importance of testing the classification in a naturalistic global setting. Early in the process, formative field studies informed the framework and direction of change at a conceptual and structural level. Evaluative field studies tested the applicability of these changes at a later stage. Field studies were conducted across the globe in around 20 field study centers, with an emphasis on diversity, culture, variability of professionals as well as variability of clinical settings [53]. The field study centers are in the following countries: Lebanon, Mexico, South Africa, India, Japan, China, Germany, Spain, Brazil, Russia, Nigeria, Italy, and Canada.

\section{The Arab world}

The Arab world includes the 22 members of the Arab League, which together consists of 280 million individuals; it is home to the biggest proportion of youth globally, as $38 \%$ of Arabs are under the age of 14 [45]. While an estimated 100-140 million people in this region suffer from at least one psychiatric disorder (World Health Organization), there remains a big gap in terms of availability of psychiatrists and other trained mental health professionals such as psychiatric nurses and psychologists [45], perhaps especially in rural areas. Wide discrepancies exist across countries of the Arab league. Qatar, Bahrain, and Kuwait have the highest number of psychiatrists per 100,000 persons, compared to Iraq, Libya, Morocco, Somalia, Sudan, Syria, and Yemen where there are fewer than 0.5 psychiatrists for every 100 , 000 persons [45].

A large number of Arab countries have consented in principle to the incorporation of mental health into the 
primary health care system; however, the application and execution of this have so far been constrained and the fundamental mental health structure as well as its services in most countries remains largely inadequate and unable to meet the increasing needs [45, 65]. For example, psychotropic medications remain unavailable in the primary care setting, due to shortages in supply, problems in distribution, cost, and poor quality of the drugs [46].

Furthermore, six of twenty countries in the region have no mental health legislation, and two, Djibouti and Lebanon, have no mental health policy [45]. The Arab region is also particular with respect to cultural factors such as stigma regarding those who have mental illness [57], and social factors which continue to hinder identification of those in need and their access to specialist care [35, 43, 47]. In terms of financing, overall, most Arab countries channel less than $2 \%$ of their health care budget towards mental health, with some contributing negligible amounts or none for mental health $[68,69]$.

\section{Main text \\ Mood disorders in ICD-10 \& ICD-11}

In ICD-10, mood, anxiety, and stress-related disorders are classified into two distinct categories: mood (affective) disorders (F30-F39) and neurotic, stress-related and somatoform disorders (F40-F48, [67]).

Mood and anxiety disorders are the most common diagnoses used in primary care and specialist settings [2]. Depression alone is increasingly recognized as a major social and economic burden and is the leading cause of disability worldwide [72]. A number of problems have been identified with this class of disorders in the 10th version of the ICD, including a disproportionate number of co-morbid depression and anxiety presentations as well as a high prevalence of somatization and the question of sensitivity to subthreshold clinical cases [2].

Cultural variations have been confirmed through the findings of a number of international studies: the WHO Collaborative Study on Standardized Assessment of Depressive Disorder and the WHO Collaborative Study on Psychological Problems in General Health Care (PPGHC). On the other hand, early myths of radical differences in the expression of distress and associated feelings were also dispelled. The focus on core symptoms seems to confirm a unitary clinical presentation of mood disorders that is affected by external factors, such as the nature of healthcare provision [14].

The ICD-11 working group on the classification of Mood and Anxiety Disorders was responsible for the development of these categories and reported to the International Advisory Group for the revision of ICD-10 Mental and Behavioral Disorders. It was chaired by Prof. Mario Maj and including a membership of experts from over 14 countries supported by additional consultants [40]. The World Psychiatric Association also actively supported the various activities, including the dissemination of information [64].

A number of changes were made with respect to this category of disorders. Since a comprehensive review is beyond the scope of this article, only the most significant changes will be explored in detail.

In ICD-11, the exclusion of a diagnosis of depressive episode in the context of bereavement was maintained, as long as the grieving process is consistent with the individual's religious and cultural context. This is a major departure from the stance adopted by the Diagnostic and Statistical Manual, 5th edition, where the exclusion has been removed altogether. While there is often not enough to differentiate these two constructs clinically on the basis of symptomatology alone [75], a strong argument in favor of keeping the exclusion is that individuals who experience a depressive reaction following a bereavement have a risk of later depression similar to the general population. It is well established that an episode of nonbereavement-related depression increases chances of experiencing a future relapse, irrespective of circumstances and adverse life events. Nonetheless, in a clinical setting, the etiological factors of a depressive illness should continue to be examined, as they remain relevant in choosing the right treatment and eliminating contributing factors [40].

In relation to bipolar affective disorder (BPAD), the changes in ICD-11 are in line with those found in Diagnostic and Statistical Manual, 5th edition. "Increased Activity or Energy" will now be a prerequisite in the criterion A for the diagnosis of hypomania and mania. According to studies, this state of psychomotor activation is almost always present in tandem with hyperthermia [60]. On a separate note, a manic or hypomanic episode emerging during antidepressant treatment and persisting beyond the immediate effect of treatment will continue to lead to a diagnosis of bipolar affective disorder, without the need for a separate iatrogenic category. No other changes are being proposed. In contrast to the Diagnostic and Statistical Manual, 5th edition, the ICD-11 will retain the category of "mixed episode". It will however be made flexible enough to encompass stable and unstable mixed states. This includes clinical cases in which the two mood states (depression and mania) are present within one episode at syndromic level as well as those in which one of the states is predominant and the other sub-syndrome [51].

It is well known that depression and anxiety are frequently comorbid $[28,58,73]$. In ICD-11, as in the Diagnostic and Statistical Manual, 5th edition, it will be possible to record the presence of sub-syndromic anxiety in a patient diagnosed with a major depressive episode, by using a specifier [21]. A specifier is "generally used to indicate notable clinical features of a disorder that may 
have prognostic, treatment, or etiological implications but are not presumed to represent a different underlying etiology or pathophysiology [21]. Mixed anxiety and depressive disorder, is now named mixed depressive and anxiety disorder and has been switched to the depressive disorders category to describe subthreshold depressive illness which has features of anxiety [25].

In ICD-11, as in the Diagnostic and Statistical Manual, 5th edition, the "Psychosis" specifier, which was a diagnostic option to consider in those with severe depression (F32.3), will be uncoupled from the dimensional specifier of severity in the diagnosis of a depressive episode; and mood episodes whether depressive, mixed, or manic may be reported to occur with or without psychotic symptoms [51].

A novel anxious depression diagnosis which is distinguished by both depressive and anxious features for a duration of 2 weeks is put forth for the classification system of mental disorders for primary care for ICD-11, the ICD-11 primary health care version (ICD-11-PHC) [74]. This is particularly important in primary care settings where patients who suffer from depression also have significant anxiety and tend to have more complicated trajectories with more disability and are at increased risk of suicide $[9,19,26,27,29,56]$. This is specifically relevant to the Arab countries and the Middle Eastern region, where people who suffer from mental illness are more likely to receive care from the primary care setting rather than in a specialized mental health setting [43].

\section{Anxiety and fear-related disorders in ICD-11}

In ICD-10, anxiety disorders are classified under neurotic, stress-related, and somatoform disorders [67], while ICD11 has shifted them to be grouped together in a novel category under anxiety and fear-related disorders [37].

This section incorporates disorders with the primary core symptoms of fear and anxiety that have to be severe enough to cause significant distress for the individual and/or impairment in functioning at a social, occupational, or educational level among others. Regardless of the source of apprehension or the feared subject/object, which form the primary basis for differentiation between them, the disorders share phenomenological similarities. These can be physiological such as increased heart rate, excessive sweating, and hyperventilation or behavioral such as escape and avoidance. A major step towards maximizing the clinical utility of the ICD-11 is to describe clearly the common core symptoms of the disorders in the grouping and to utilize this as a means to produce their "essential features" in a uniform way [37]. Therefore, a required features section relates the nature of the anxiety/fear as pertaining to a specific "focus of apprehension", which is the element or the situation that provokes the anxiety/fear [37]. The ICD-11 also emphasizes the wide range of possible emotional responses which may include shame, anger, or disgust $[15,20,30,31,37,55]$.

There is no longer a distinction between phobic anxiety disorders from other anxiety disorders as was present in ICD-10, rather ICD-11 references in addition to anxiety, fear in the title of the grouping [37]. Agoraphobia is kept as a separate diagnostic entity, and a panic attack specifier may be applied if the panic attacks occur only in response to exposure to agoraphobic situations [37]. Importantly in ICD-11, the essential symptom of agoraphobia is severe and disproportionate fear or anxiety that happens in or as a response to the anticipation of several scenarios where escape may be hard or where help may not be obtainable., this is different from the restricted ICD-10 notion of "fear of open spaces and related situations such as crowds where an escape to a safe place may be difficult" [37]. This is important so as not to restrict the situations which may illicit a fear response and to account for anxiety-provoking situations that may be reported in low- and middle-income countries [59]. What is more, the addition of a cognitive element to the description of agoraphobia., may in turn help increase this diagnosis' reliability, especially with respect to differentiating it from specific phobia [37] to note that ICD-11 proposes to omit specific subtypes of specific phobia [17],in line with its efforts to avoid over-specifications that have no treatment repercussions [22].

Social anxiety disorder (SAD)-previously social phobias in ICD-10-again follows the pattern above whereby the specific focus of apprehension in this case is the worry that the patient will behave in a fashion that will be negatively appraised by other people [17]. Further, ICD gives information that will aid in distinguishing SAD from non-pathologic agecongruent fears as well as to differentiate it from the normal emotion of fear in social contexts and the trait of shyness; the latter is largely related to the extent of impairment and distress [17].

Lastly, for generalized anxiety disorder (GAD), ICD11 proposes that the symptoms last for "several" months rather than specifying a specific duration criterion, and gives information to help distinguish normal reactions to stress from GAD or from adjustment disorder [37].

\section{A new category of stress-related disorders in ICD-11}

The etiology of a mental disorder is often unknown or is speculated to be due to a combination of factors, and in particular an interplay between genetic predisposing factors and environmental ones [62]. An identifiable causative factor is not necessary for the purpose of a diagnosis except when substances or organic causes are involved. Stress-related disorders seem to be the other exception 
despite difficulties in defining or measuring stress, while in some cases, isolated events perceived consensually as traumatic can be identified, in most situations, "stressful" triggers leading up to a psychiatric disorder are more subtle and cumulative.

The two most commonly used stress-related diagnoses are adjustment disorder [18] and post-traumatic stress disorder (PTSD) [38]. This category of diagnoses is crucial and especially relevant in the Arab region, where war outbreaks, violence, discrimination, poverty, and other risk factors for trauma are ongoing and have devastating repercussions and produce a very large number of refugees whose needs are not met [12]. It is also important to keep in mind that such conflicts have mental health consequences spanning many years and affecting several generations [24, 34, 41, 42].

In the Arab region, a big proportion of those who suffer from trauma, both in terms of physical and psychological symptoms present to the primary care sector [46]. What is more, apart from war-related trauma, a large number of patients in this region face social problems, which increase their risk for mental disorders or represent in themselves obstacles to care. Some are common to western contexts such as separation or divorce and other more specific to the political instability in the region such as refugee status [46]. Child abuse, elder abuse, and sexual assault seem to be forms of violence that are more common than others in the Arab region [46].

In ICD-10, stress and trauma are recognized as etiological factors in a number of disorders such as acute stress disorder, adjustment disorder, post-traumatic stress disorder (F43), and enduring personality change after traumatic experience (F62). This is also the case for disorders and conditions not included in the category of anxiety and stress-related disorders, such as reactive attachment disorder in childhood and disinhibited attachment disorder in childhood (F94). Chapter 21 of the ICD-10 allows a clinician to make note the effect of adverse life events, such as the death of a family member [67]. Instead, the ICD-11 is for a lumping of the relevant diagnoses into one category of disorders specifically associated with stress.

The use of the diagnosis of PTSD has been a topic of controversy, and there has been criticism over its wide array of symptom clusters and number of possible presentations, as well as the symptom overlap with other psychiatric illnesses $[10,39]$. In contrast to the direction taken by the Diagnostic and Statistical Manual, 5th edition, the ICD-11 working group chose to adopt the narrower more focused definition of PTSD by reinforcing the role of the set of criteria specific to the condition, whereby the presence of avoidance, re-experiencing, and hypervigilance to a recognized threat are the core symptoms [38]. In parallel, ICD-11 proposes the creation of an all-new diagnosis of complex PTSD, where the disturbances that follow the trauma are noted at a more pervasive level, and this diagnosis incorporates symptoms in three clusters: symptoms that are intra- and interpersonal as well as the core symptoms of PTSD [38].

Another new diagnosis is that of prolonged grief disorder, which is characterized by an intense, prolonged, and disabling grief beyond the normative response [38]. Acute stress reaction was deemed to be non-pathological given its often proportional and time-limited response to exceptional event. As such, the proposal is to move it to the Z-Chapter in ICD-11 [38].

Using the ICD-11 Global Clinical Practice Network, mental health professionals were selected to test the validity and reliability of already established stress-related disorders in a series of clinical vignettes [36]. These were followed by field studies involving real patients. They revealed an improved inter-clinician reliability for some diagnoses but not all [53]. A diagnosis of adjustment disorder often has serious consequences in particular, it is highly associated with suicidality [13] and seems to be very prevalent after traumatic injuries [44]. ICD-11 has included clearly defined symptoms and exclusion criteria for Adjustment Disorder whereby symptoms should not have the severity of any other mental disorder, as is also the case in the Diagnostic and Statistical Manual, 5th edition. The modified adjustment disorder diagnosis with the abolition of the subtypes and tightening of the criteria shows significantly better reliability ( $73 \%$ vs $54 \%$ ) according to the results of the field studies. On the other hand, the Kappa measure for a PTSD diagnosis dropped from $62 \%$ in ICD-10 to $49 \%$ in ICD-11 [53]. This seems to reflect the complexity of this diagnosis and the difficulties in conceptualizing it consistently across cultures and settings.

\section{Implications for the Arab region}

The field trials have been completed internationally. The next step involves the implementation of ICD-11 in member countries and training of clinicians on this new classification system [71]. In the Arab region, mental health training programs for primary care physicians, non-physicians, and health personnel working at the level of primary health care have begun as a part of inservice skills enhancement programs [65]. However, mental health care is still lagging in terms of the human resources required to expand and develop services beyond provision for the most severely ill $[43,46]$.

Nevertheless, there are some positive steps in psychiatric services, which have been restricted to a small number of mental hospitals, are slowly being exchanged to specialized psychiatric units that provide inpatient and outpatient care and some have even decentralized care to provide psychiatric services at district hospitals 
and small peripheral units [45]. Such developments should allow better access to mental health care for sections of the population in underserved and rural areas. Having a locally validated and reliable classification system acquires even more importance in this context.

\section{Conclusion}

The primary aim backing the revision remains to improve clinical utility from a public health standpoint [32]. The global emphasis of the field trials provides a unique chance for a truly international and multidisciplinary collaboration.

With the promise of an ongoing update mechanism of the ICD-11 even after its publication, the contribution of clinicians is more likely than ever before to influence the direction taken by classification systems for the coming decades. This, in tandem with the mental health global action program, a World Health Organization initiative [66], will inform policy and impact practice at international and more local levels.

The ICD-11 constitutes an extensive revision of an important classification system for mental illnesses. The revision process recruited a variety of clinicians and experts in the mental health field and necessitated unparalleled cooperation on a global and international scale. Up to date, evidence was taken into account to promote the validity of the International Classification of Diseases and to make sure it is suitable and appropriate for application in various regions in the world [51].

\begin{abstract}
Abbreviations
BPAD: Bipolar affective disorder; DSM: Diagnostic and statistical manual of mental disorders; GAD: Generalized anxiety disorder; ICD: International Classification of Diseases; ICD-10: International Classification of Diseases, 10th edition; ICD-10-PHC: International Classification of Diseases Primary Health Care, 10th edition; ICD-11: International Classification of Diseases, 11th edition; ICD-11-PHC: International Classification of Diseases Primary Health Care, 11th edition; OCD: Obsessive-compulsive disorder; PTSD: Post traumatic stress disorder; SAD: Social anxiety disorder; WHO: World Health Organization
\end{abstract}

\section{Acknowledgements}

None

\section{Authors' contributions}

EB and JEK contributed to the literature review and first draft of the paper. BK contributed to adding sections to and revising the drafted manuscript. All authors read and approved the final manuscript.

\section{Funding}

None

Availability of data and materials

Not applicable

\section{Ethics approval and consent to participate}

Not applicable

\section{Consent for publication}

Not applicable

\section{Competing interests}

The authors declare that they have no competing interests.
Received: 5 November 2019 Accepted: 14 January 2020

Published online: 25 February 2020

\section{References}

1. (2013) American Psychiatric Association: diagnostic and statistical manual of mental disorders, Fifth. American Psychiatric Association

2. Andrade L, Wang YP (2012) How global epidemiological evidence can inform the revision of ICD-10 classification of depression 6 and anxiety disorders. World Psychiatry:6-11

3. Ayuso-Mateos JL (2012) Prototype diagnosis of psychiatric syndromes and the ICD-11. World Psychiatry 11:30-31

4. Baca-Garcia E, Perez-Rodriguez MM, Basurte-Villamor I et al (2007) Diagnostic stability of psychiatric disorders in clinical practice. Br J Psychiatry 190:210-216 https://doi.org/10.1192/bjp.bp.106.024026

5. Bandelow B, Reitt M, Röver C et al (2015) Efficacy of treatments for anxiety disorders: a meta-analysis. Int Clin Psychopharmacol 30:183-192 https://doi. org/10.1097/YIC.0000000000000078

6. Basílio N, Ramos C, Figueira S, Pinto D (2016) Worldwide usage of International Classification of Primary Care. Rev Bras Med Família e Comunidade 11:1-9 https://doi.org/10.5712/rbmfc11(38)1225

7. Biswas J, Gangadhar BN, Keshavan M (2016) Cross cultural variations in psychiatrists' perception of mental illness: a tool for teaching culture in psychiatry. Asian J Psychiatr 23:1-7 https://doi.org/10.1016/j.ajp.2016.05.011

8. Blier P (2005) Atypical antipsychotics for mood and anxiety disorders: safe and effective adjuncts? J Psychiatry Neurosci 30:232-233

9. Bomyea J, Lang AJ, Craske MG et al (2013) Suicidal ideation and risk factors in primary care patients with anxiety disorders. Psychiatry Res 209:60-65 https://doi.org/10.1016/.j.psychres.2013.03.017

10. Brewin CR, Lanius RA, Novac A et al (2009) Reformulating PTSD for DSM-V: life after Criterion A. J Trauma Stress 22:366-373 https://doi.org/ $10.1002 / j t s .20443$

11. Carlsen HK, Steingrimsson S, Sigurdsson MI, Magnusson A (2015) The stability of diagnosis over time in a register-based study of psychiatric inpatients. Eur Psychiatry 30:1385 https://doi.org/10.1016/S09249338(15)32037-X

12. Carta MG, Moro MF, Bass J (2015) War traumas in the Mediterranean area. Int J Soc Psychiatry 61:33-38 https://doi.org/10.1177/0020764014535754

13. Casey P, Jabbar F, O'Leary E, Doherty AM (2015) Suicidal behaviours in adjustment disorder and depressive episode. J Affect Disord 174:441-446 https://doi.org/10.1016/j.jad.2014.12.003

14. Chakrabarti S, Berlanga C, Njenga F (2012) Cultural issues in the classification and diagnosis of mood and anxiety disorders. World Psychiatry:26-31

15. Cisler JM, Olatunji BO, Lohr JM (2009) Disgust, fear, and the anxiety disorders: a critical review. Clin Psychol Rev 29:34-46 https://doi.org/10. 1016/j.cpr.2008.09.007

16. Craighead WE, Sheets E, Brosse A, llardui S (2007) Psychosocial treatments for major depressive disorder. In: Nathan P, Gorman J (eds) A Guide To Treatments That Work. Oxford University

17. Emmelkamp PMG (2012) Specific and social phobias in ICD-11. World Psychiatry 11

18. Evans SC, Reed GM, Roberts MC et al (2013) Psychologists' perspectives on the diagnostic classification of mental disorders: results from the WHOIUPsyS Global Survey. Int J Psychol 48:177-193 https://doi.org/10.1080/ 00207594.2013.804189

19. Fava M, Rush AJ, Alpert JE et al (2008) Difference in treatment outcome in outpatients with anxious versus nonanxious depression: a STAR*D report. Am J Psychiatry 165:342-351 https://doi.org/10.1176/appi.ajp.2007.06111868

20. Fergus $T A$, Valentiner DP, McGrath PB, Jencius S (2010) Shame- and guiltproneness: relationships with anxiety disorder symptoms in a clinical sample. J Anxiety Disord 24:811-815 https://doi.org/10.1016/j.janxdis.2010. 06.002

21. First MB (2012) Challenges in the implementation of diagnostic specifiers for mood disorders in ICD-11. World Psychiatry

22. First MB, Reed GM, Hyman SE, Saxena S (2015) The development of the ICD-11 Clinical Descriptions and Diagnostic Guidelines for Mental and Behavioural Disorders. World Psychiatry 14:82-90 https://doi.org/10.1002/ wps. 20189

23. Gaebel W, Zielasek J, Reed G (2017) Mental and behavioural disorders in the ICD-11: concepts, methodologies, and current status. Psychiatr Pol 51:169195 https://doi.org/10.12740/PP/69660 
24. Ginzburg K, Ein-Dor T, Solomon Z (2010) Comorbidity of posttraumatic stress disorder, anxiety and depression: a 20-year longitudinal study of war veterans. J Affect Disord 123:249-257 https://doi.org/10.1016/j.jad. 2009.08.006

25. Goldberg D (2012) The overlap between the common mental disorders challenges for classification. Int Rev Psychiatry 24:549-555 https://doi.org/10. 3109/09540261.2012.742041

26. Goldberg D, Fawcett J (2012) The importance of anxiety in both major depression and bipolar disorder. Depress Anxiety 29:471-478 https://doi org/10.1002/da.21939

27. Goldberg DP (2014) Anxious forms of depression. Depress Anxiety 31:344351 https://doi.org/10.1002/da.22206

28. Goldberg DP, Prisciandaro JJ, Williams P (2012) The primary health care version of ICD-11: the detection of common mental disorders in general medical settings. Gen Hosp Psychiatry 34:665-670 https://doi.org/10.1016/j. genhosppsych.2012.06.006

29. Goldberg DP, Wittchen H-U, Zimmermann P et al (2014) Anxious and nonanxious forms of major depression: familial, personality and symptom characteristics. Psychol Med 44:1223-1234 https://doi.org/10.1017/ S0033291713001827

30. Hawkins KA, Cougle JR (2011) Anger problems across the anxiety disorders: findings from a population-based study. Depress Anxiety 28:145-152 https://doi.org/10.1002/da.20764

31. Hedman E, Ström P, Stünkel A, Mörtberg E (2013) Shame and guilt in social anxiety disorder: effects of cognitive behavior therapy and association with social anxiety and depressive symptoms. PLoS One 8:e61713 https://doi.org/ 10.1371/journal.pone.0061713

32. International Advisory Group for the Revision of ICD-10 Mental and Behavioural Disorders (2011) A conceptual framework for the revision of the ICD-10 classification of mental and behavioural disorders. World Psychiatry 10:86-92

33. Kaczkurkin AN, Foa EB (2015) Cognitive-behavioral therapy for anxiety disorders: an update on the empirical evidence. Dialogues Clin Neurosci 17: 337-346

34. Karam EG, Fayyad J, Nasser Karam A et al (2008) Effectiveness and specificity of a classroom-based group intervention in children and adolescents exposed to war in Lebanon. World Psychiatry 7:103-109

35. Karam EG, Mneimneh ZN, Karam AN et al (2006) Prevalence and treatment of mental disorders in Lebanon: a national epidemiological survey. Lancet 367:1000-1006 https://doi.org/10.1016/S0140-6736(06)68427-4

36. Keeley JW, Reed GM, Roberts MC et al (2016) Disorders specifically associated with stress: a case-controlled field study for ICD-11 mental and behavioural disorders. Int J Clin Heal Psychol 16:109-127 https://doi.org/10. 1016/j.ijchp.2015.09.002

37. Kogan CS, Stein DJ, Maj M et al (2016) The classification of anxiety and Fearrelated disorders in the ICD-11. Depress Anxiety 33:1141-1154 https://doi. org/10.1002/da.22530

38. Maercker A, Brewin CR, Bryant RA et al (2013) Proposals for mental disorders specifically associated with stress in the International Classification of Diseases-11. Lancet 381:1683-1685 https://doi.org/10.1016/S01406736(12)62191-6

39. Maercker A, Brewin CR, Bryant RA et al (2013) Diagnosis and classification of disorders specifically associated with stress: proposals for ICD-11. World Psychiatry 12:198-206 https://doi.org/10.1002/wps.20057

40. Maj M, Reed GM (2012) The development of the ICD-11 classification of mood and anxiety disorders. World Psychiatry 11:3-6

41. Mollica RF, Caridad KR, Massagli MP (2007) Longitudinal study of posttraumatic stress disorder, depression, and changes in traumatic memories over time in Bosnian refugees. J Nerv Ment Dis 195:572-579 https://doi.org/10.1097/NMD.0b013e318093ed2c

42. Mollica RF, Sarajlic N, Chernoff M et al (2001) Longitudinal study of psychiatric symptoms, disability, mortality, and emigration among Bosnian refugees. JAMA 286:546-554

43. Nasser SC, Salamoun MM (2011) Treatment of mental disorders and pathways to care in Arab countries. Int J Psychiatry Clin Pract 15:12-18 https://doi.org/10.3109/13651501.2010.512664

44. O'Donnell ML, Alkemade N, Creamer M et al (2016) A longitudinal study of adjustment disorder after trauma exposure. Am J Psychiatry 173:1231-1238 https://doi.org/10.1176/appi.ajp.2016.16010071

45. Okasha A, Karam E, Okasha T (2012) Mental health services in the Arab world. World Psychiatry 11:52-54
46. Osman OT, Nasir L, Mollica RF, et al (2017) Trauma-informed care survey of psychiatrists and primary care physicians in the Middle East. Prim Care Companion CNS Disord 19:. https://doi.org/10.4088/PCC. $17 \mathrm{~m} 02157$

47. Patel V (2007) Mental health in low- and middle-income countries. Br Med Bull 81-82:81-96 https://doi.org/10.1093/bmb/ldm010

48. Patel V (2014) Why mental health matters to global health. Transcult Psychiatry 51:777-789 https://doi.org/10.1177/1363461514524473

49. Rathod S, Pinninti N, Irfan M et al (2017) Mental health service provision in low- and middle-income countries. Heal Serv insights 10: $1178632917694350 \mathrm{https}: / / d o i . o r g / 10.1177 / 1178632917694350$

50. Reed GM (2010) Toward ICD-11: improving the clinical utility of WHO's International Classification of mental disorders. Prof Psychol Res Pract 41: 457-464 https://doi.org/10.1037/a0021701

51. Reed GM, First MB, Kogan CS et al (2019) Innovations and changes in the ICD-11 classification of mental, behavioural and neurodevelopmental disorders. World Psychiatry 18:3-19 https://doi.org/ 10.1002/wps.20611

52. Reed GM, Mendonça Correia J, Esparza P et al (2011) The WPA-WHO global survey of psychiatrists' attitudes towards mental disorders classification. World Psychiatry 10:118-131

53. Reed GM, Sharan P, Rebello TJ et al (2018) The ICD-11 developmental field study of reliability of diagnoses of high-burden mental disorders: results among adult patients in mental health settings of 13 countries. World Psychiatry 17:174-186 https://doi.org/10.1002/wps.20524

54. (2007) Scale up services for mental disorders: a call for action. Lancet 370: 1241-1252. https://doi.org/10.1016/S0140-6736(07)61242-2

55. Schoenleber M, Chow PI, Berenbaum H (2014) Self-conscious emotions in worry and generalized anxiety disorder. Br J Clin Psychol 53:299-314 https:// doi.org/10.1111/bjc.12047

56. Seo H-J, Jung Y-E, Kim T-S et al (2011) Distinctive clinical characteristics and suicidal tendencies of patients with anxious depression. J Nerv Ment Dis 199:42-48 https://doi.org/10.1097/NMD.0b013e3182043b60

57. Sewilam AM, Watson AM, Kassem AM et al (2015) Suggested avenues to reduce the stigma of mental illness in the Middle East. Int J Soc Psychiatry 61:111-120 https://doi.org/10.1177/0020764014537234

58. Simms LJ, Prisciandaro JJ, Krueger RF, Goldberg DP (2012) The structure of depression, anxiety and somatic symptoms in primary care. Psychol Med 42 : 15-28 https://doi.org/10.1017/S0033291711000985

59. Stein DJ (2012) Agoraphobia and panic disorder: options for ICD-11. World Psychiatry

60. Strakowski S (2012) Bipolar disorders in ICD-11. World Psychiatry:31-36

61. Thornicroft G, Chatterji S, Evans-Lacko S et al (2017) Undertreatment of people with major depressive disorder in 21 countries. Br J Psychiatry 210: 119-124 https://doi.org/10.1192/bjp.bp.116.188078

62. Uher R (2014) Gene-environment interactions in common mental disorders: an update and strategy for a genome-wide search. Soc Psychiatry Psychiatr Epidemiol 49:3-14 https://doi.org/10.1007/s00127-013-0801-0

63. Üstun TB, Sartorius N (1994) ICD-10 primary care version for recognition and management of mental disorders. Past, Present and Future of Psychiatry. WORLD SCIENTIFIC, In, pp 179-184

64. Volpe U (2013) WPA contribution to the development of the chapter on mental disorders of the ICD-11: an update. World Psychiatry 12:183-184 https://doi.org/10.1002/wps.20048

65. WHO (2006) Mental health in the Eastern Mediterranean Region reaching the unreached

66. WHO, UNHCR (2015) mhGAP humanitarian intervention guide (mhGAP-HIG) clinical management of mental, neurological and substance use conditions in humanitarian emergencies

67. World Health Organization (1992) International classification of diseases and related health problems, 10 th revision.

68. World Health Organization (2010) Maternal, child and adolescent mental health: challenges and strategic directions 2010-2015

69. World Health Organization (2010) Mental health systems in the Eastern Mediterranean Region

70. World Health Organization (2018) WHO mental health gap action programme. https://www.who.int/mental_health/mhgap/en/

71. World Health Organization (2019) ICD-11 implementation or transition guide. ICD-11 Implement or Transit Guid 1-29

72. (2018) World Health Organization fact sheets. https://www.who.int/newsroom/fact-sheets/detail/depression 
73. Zbozinek TD, Rose RD, Wolitzky-Taylor KB et al (2012) Diagnostic overlap of generalized anxiety disorder and major depressive disorder in a primary care sample. Depress Anxiety 29:1065-1071 https:/doi.org/10.1002/da.22026

74. Ziebold C, Mari JJ, Goldberg DP et al (2019) Diagnostic consequences of a new category of anxious depression and a reduced duration requirement for anxiety symptoms in the ICD-11 PHC. J Affect Disord 245:120-125 https://doi.org/10.1016/j.jad.2018.10.082

75. Zisook S, Corruble E, Duan N et al (2012) The bereavement exclusion and DSM-5. Depress Anxiety 29:425-443 https://doi.org/10.1002/da.21927

\section{Publisher's Note}

Springer Nature remains neutral with regard to jurisdictional claims in published maps and institutional affiliations.

\section{Submit your manuscript to a SpringerOpen ${ }^{\circ}$ journal and benefit from:}

- Convenient online submission

- Rigorous peer review

- Open access: articles freely available online

- High visibility within the field

- Retaining the copyright to your article

Submit your next manuscript at $\boldsymbol{\sim}$ springeropen.com 